\title{
Molecular Fluorescence Endoscopy Targeting Vascular Endothelial Growth Factor A for Improved Colorectal Polyp Detection
}

\author{
Jolien J. Tjalma*1, P. Beatriz Garcia-Allende*2, Elmire Hartmans ${ }^{1}$, Anton G. Terwisscha van Scheltinga ${ }^{3}$, \\ Wytske Boersma-van Ek ${ }^{1}$, Jürgen Glatz ${ }^{2}$, Maximilian $\mathrm{Koch}^{2}$, Yasmijn J. van Herwaarden ${ }^{4}$, Tanya M. Bisseling ${ }^{4}$, \\ Iris D. Nagtegaal ${ }^{5}$, Hetty Timmer-Bosscha ${ }^{6}$, Jan Jacob Koornstra ${ }^{1}$, Arend Karrenbeld ${ }^{7}$, Jan H. Kleibeuker ${ }^{1}$, \\ Gooitzen M. van Dam ${ }^{8}$, Vasilis Ntziachristos ${ }^{2}$, and Wouter B. Nagengast ${ }^{1}$ \\ ${ }^{1}$ Department of Gastroenterology and Hepatology, University of Groningen, University Medical Center Groningen, Groningen, \\ The Netherlands; ${ }^{2}$ Chair for Biological Imaging \& Institute for Biological and Medical Imaging, Technical University of Munich and \\ Helmholtz Center Munich, Munich, Germany; ${ }^{3}$ Department of Clinical Pharmacy and Pharmacology, University of Groningen, \\ University Medical Center Groningen, Groningen, The Netherlands; ${ }^{4}$ Department of Gastroenterology and Hepatology, Radboud \\ University Medical Center, Nijmegen, The Netherlands; ${ }^{5}$ Department of Pathology, Radboud University Medical Center, Nijmegen, \\ The Netherlands; ${ }^{6}$ Department of Medical Oncology, University of Groningen, University Medical Center Groningen, Groningen, \\ The Netherlands; ${ }^{7}$ Department of Pathology, University of Groningen, University Medical Center Groningen, Groningen, The \\ Netherlands; and ${ }^{8}$ Department of Surgery, University of Groningen, University Medical Center Groningen, Groningen, \\ The Netherlands
}

Small and flat adenomas are known to carry a high miss-rate during standard white-light endoscopy. Increased detection rate may be achieved by molecular fluorescence endoscopy with targeted nearinfrared (NIR) fluorescent tracers. The aim of this study was to validate vascular endothelial growth factor $A$ (VEGF-A) and epidermal growth factor receptor (EGFR)-targeted fluorescent tracers during ex vivo colonoscopy with an NIR endoscopy platform. Methods: VEGF-A and EGFR expression was determined by immunohistochemistry on a large subset of human colorectal tissue samples-48 sessile serrated adenomas/polyps, 70 sporadic high-grade dysplastic adenomas, and 19 hyperplastic polyps-and tissue derived from patients with Lynch syndrome-78 low-grade dysplastic adenomas, 57 high-grade dysplastic adenomas, and 31 colon cancer samples. To perform an ex vivo colonoscopy procedure, 14 mice with small intraperitoneal EGFR-positive HCT116luc tumors received intravenous bevacizumab-800CW (anti-VEGF-A), cetuximab-800CW (anti-EGFR), control tracer IgG-800CW, or sodium chloride. Three days later, 8 resected HCT116luc tumors $(2-5 \mathrm{~mm})$ were stitched into 1 freshly resected human colon specimen and followed by an ex vivo molecular fluorescence colonoscopy procedure. Results: Immunohistochemistry showed high VEGF-A expression in $79 \%-96 \%$ and high EGFR expression in $51 \%-69 \%$ of the colorectal lesions. Both targets were significantly overexpressed in the colorectal lesions, compared with the adjacent normal colon crypts. During ex vivo molecular fluorescence endoscopy, all tumors could clearly be delineated for both bevacizumab-800CW and cetuximab-800CW tracers. Specific tumor uptake was confirmed with fluorescent microscopy showing, respectively, stromal and cell membrane fluorescence. Conclusion: VEGF-A is a promising target for molecular fluorescence endoscopy because it showed a high protein expres-

\footnotetext{
For correspondence or reprints contact: Wouter B. Nagengast, Department of Gastroenterology and Hepatology, BB41, University Medical Center Groningen, P.O. Box 30.001, 9700 RB Groningen, The Netherlands.

E-mail: w.b.nagengast@umcg.nl

${ }^{*}$ Contributed equally to this work.

Published online Dec. 17, 2015.

COPYRIGHT (C) 2016 by the Society of Nuclear Medicine and Molecular Imaging, Inc.
}

sion, especially in sessile serrated adenomas/polyps and Lynch syndrome. We demonstrated the feasibility to visualize small tumors in real time during colonoscopy using a NIR fluorescence endoscopy platform, providing the endoscopist a wide-field redflag technique for adenoma detection. Clinical studies are currently being performed in order to provide in-human evaluation of our approach.

Key Words: optical imaging; molecular imaging; vascular endothelial growth factor A; near-infrared fluorescence; endoscopy

\section{J Nucl Med 2016; 57:480-485}

DOI: 10.2967/jnumed.115.166975

$\mathbf{W}$

hite-light endoscopy is the gold standard for detection of premalignant and malignant colorectal lesions (1). Despite the efficacy of current colonoscopy, small and especially right-sided flat or serrated adenomas are notoriously difficult to detect, resulting in substantial polyp detection miss-rates of $20 \%-26 \%(2,3)$. Missed lesions are a main risk factor for the occurrence of interval cancers, particularly in high-risk patients such as patients with Lynch syndrome (LS) (4-6). Advanced endoscopic imaging modalities, such as narrow-band imaging, autofluorescence imaging, and chromoendoscopy, have been extensively investigated but did not show significant improvement in adenoma detection rates (7-9). Ideally, white-light endoscopy is combined with a sensitive, wide-fieldof-view, red-flag technique to assist the endoscopist in the immediate identification of aberrant lesions. Molecular optical imaging, that is, visualizing the molecular signature of cells in vivo, would be highly suitable for this aim. It enables real-time anatomic and functional imaging, and it is safe, fast, and relatively inexpensive (10). With selective optical agents functioning in the near-infrared (NIR) light spectrum, contrast between normal mucosa and dysplastic tissue could potentially be greatly enhanced, thereby reducing miss-rates (11). These agents should target biomarkers known to 
be overexpressed in colorectal cancer such as epidermal growth factor receptor (EGFR) or shown to be overexpressed early in the adenomacarcinoma sequence, as described for vascular endothelial growth factor A (VEGF-A), due to the angiogenic switch $(12,13)$. The aim of the current study was to validate VEGF-A- and EGFR-targeting fluorescent antibodies in visualizing small colorectal lesions in real time with our novel NIR endoscopy platform. Therefore, we first determined the expression of potential targets VEGF-A and EGFR in archival human colorectal samples, including sessile serrated adenomas/polyps (SSA/P) and adenomatous and carcinomatous tissue of patients with LS. Second, we performed a simulated endoscopy procedure to validate our VEGF-A- and EGFR-targeting NIR fluorescent tracers.

\section{MATERIALS AND METHODS}

\section{Immunohistochemistry of Human Colorectal Lesions}

The archival formalin-fixed and paraffin-embedded human colorectal tissue set consisted of SSA/P ( $n=48 ; 42$ different individuals), Lynch low-grade dysplasia (LGD) adenomas ( $n=78 ; 62$ individuals), Lynch high-grade dysplasia (HGD) adenomas ( $n=57$; 40 individuals), Lynch colon cancer tissue ( $n=32 ; 31$ individuals), sporadic adenomas with HGD ( $n=70 ; 70$ individuals), and hyperplastic polyps (HP) ( $n=19 ; 18$ individuals). The tissue was handled according to Dutch Code of Conduct for proper use of Human Tissue (www.federa.org), and the study was conducted according to the guidelines of the medical ethical committee of our hospital (www.ccmo.nl). To evaluate the clinical relevance of an anti-VEGF and anti-EGFR fluorescent tracer for colorectal surveillance, the protein expression of VEGF-A and EGFR was determined by immunohistochemistry. Slides were rehydrated via graded alcohols. Antigen was retrieved with $0.100 \mathrm{M}$ Tris $/ \mathrm{HCl}$

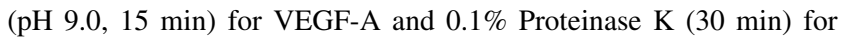
EGFR. Endogenous peroxidase blocking was performed during 30 min with $0.42 \%$ hydrogen peroxide, followed by avidin-biotin blocking. For VEGF-A, sections were incubated for $1 \mathrm{~h}$ with polyclonal rabbit antihuman VEGF-A (Santacruz) (1:50) and consecutive for $30 \mathrm{~min}$ with swine antirabbit biotin (1:300 in phosphate-buffered saline [PBS] with $1 \%$ bovine serum albumin [BSA]) and streptavidin (1:300 in PBS/1\% BSA). For EGFR, sections were incubated for $1 \mathrm{~h}$ with monoclonal mouse anti-EGFR (clone 31G7; Invitrogen) (1:50 in $\mathrm{PBS} / 1 \% \mathrm{BSA}$ ) and consecutive for $30 \mathrm{~min}$ with rabbit antimouse peroxidase (1:100) and goat antirabbit peroxidase (1:100). Color development was achieved by applying a 3-3'-diaminobenzine-tetrahydorchloride reagent (Sigma) for $10 \mathrm{~min}$. PBS was used throughout for washing, and all steps occurred at room temperature. Finally, sections were counterstained with Mayer hematoxylin, dehydrated in alcohol, and coverslipped. Staining intensities were independently evaluated by 2 individuals; consensus was achieved in discrepant cases by consulting an experienced pathologist. Dysplasia, cancer, and adjacent normal epithelium, if present, were separately scored and compared. Staining intensities were graded using a $0-3$ scale ( 0 , completely negative; 1 , weak; 2 , moderate; 3 , strong staining).

\section{Cell Culture}

The HCT116 ${ }^{\text {luc }}$ human colon cancer cell line, stably transfected with the firefly gene luciferase, was obtained from Caliper Life Sciences. The cells were grown in a monolayer culture using McCoy 5A Medium (Gibco, Life Technologies) supplemented with $10 \%$ fetal bovine serum, in a humidified atmosphere containing $5 \% \mathrm{CO}_{2}$ at $37^{\circ} \mathrm{C}$. EGFR cell surface expression was confirmed for HCT116 luc (data not shown) with fluorescence-activated cell sorting (FACS Calibur; BD Biosciences).

\section{Fluorescent Labeling of Monoclonal Antibodies}

As described previously, the monoclonal antibodies bevacizumab (Roche), cetuximab (Merck), and human IgG (Nanogram; Sanquin) were labeled with IRDye800CW-NHS (LI-COR Biosciences) $(14,15)$. This NIR fluorescent dye underwent extensive toxicity testing and is a good-manufacturing-practice-compliant compound, registered at the U.S. Food and Drug Administration (16,17).

\section{HCT116 luc Human Xenograft Tumors}

All experiments were approved by the animal welfare committee of the University of Groningen and performed in accordance with the Dutch Animal Welfare Act of 1997. To obtain multiple small tumor lesions, 14 male athymic nude mice (Harlan) received an intraperitoneal injection with $200 \mu \mathrm{L}$ of $2 \times 10^{6} \mathrm{HCT} 116^{\text {luc }}$ cells suspended in PBS. Tumor growth was monitored with intraperitoneal injections with D-luciferin reconstituted in PBS (1.5 mg in $100 \mu \mathrm{L}$; PerkinElmer), followed by bioluminesence imaging with an in vivo imaging system (IVIS Spectrum; Caliper Life Sciences). At day 14, all mice reached bioluminescent signals, indicating sufficient tumor growth, after which $100 \mu \mathrm{g}$ of bevacizumab-800CW $(n=5), 100 \mu \mathrm{g}$ of cetuximab-800CW $(n=5), 100 \mu \mathrm{g}$ of human IgG-800CW $(n=2)$, or sodium chloride $(n=2)$ were injected in a total volume of $200 \mu \mathrm{L}$. Intravenous injection was performed via the penile vein under general anesthesia. The mice receiving IgG-800CW or sodium chloride served as negative controls. At day 3 after injection, mice were euthanized by cervical dislocation and 8 small intraperitoneal tumors $(2-5 \mathrm{~mm})$ were harvested for ex vivo colonoscopy purposes ( 2 per tracer). The remaining intraperitoneal tumors (varying in size between 2 and $5 \mathrm{~mm}$ ) and mouse organs (liver, colon, and muscle) were harvested for ex vivo analyses.

\section{NIR Fluorescence Endoscopy Platform}

The NIR fluorescence endoscopy platform consists of a custommade Micrendo fiber bundle containing 30,000 coherently arranged individual fibers (Schölly Fiberoptic $\mathrm{GmbH}$ ). The imaging bundle conducts the images to the sensor module of a previously developed clinical prototype NIR camera system $(18,19)$. This camera system comprises a color camera and a monochrome one, which operate in parallel for white-light and NIR fluorescence acquisition, respectively. A beam splitter (T760lpxr; Chroma Technology) separates the color and NIR image components. Appropriate filtering is provided by a white light short-pass filter with a cutoff wavelength of $750 \mathrm{~nm}$ and a fluorescence emission bandpass filter with a central wavelength of $819 \mathrm{~nm}$ (bandwidth, $44 \mathrm{~nm}$ ) (THORLABS). Connection of the fiber bundle to the camera system is made feasible via a mechanical and focusing adapter, and a multibranched fiber optic bundle (SEDI-ATI Fibres Optiques) realizes simultaneous white-light illumination and fluorescence excitation (laser at $750 \mathrm{~nm}$ ) coupling. The performance of the clinical endoscopy platform, namely the characterization of the optical resolution and the sensitivity, was determined in the same manner as for the preclinical platform (20).

\section{Simulation of NIR Molecular Fluorescence Endoscopy Procedure}

To simulate a clinical colonoscopy procedure, an 11-cm-long human colon specimen was derived from a right hemicolectomy of a patient with colon cancer (requirement to obtain informed consent was waived by the Medical Ethical Committee Groningen, METC nr. 2013.446). Immediately after the surgical resection, the healthy part of the colon specimen was transported to the endoscopy suite. Two freshly resected HCT116 ${ }^{\text {luc }}$ intraperitoneal tumors $(2-5 \mathrm{~mm}$ in diameter) per tracer (bevacizumab-800CW, cetuximab-800CW, IgG-800CW, or sodium chloride) were stitched onto the luminal side of the colon wall (Fig. 1). Molecular fluorescence endoscopy was performed using a clinical video endoscope (Olympus Exera II GIF-180 series; Olympus), with the fiber bundle of the NIR fluorescence endoscopy platform inserted through the working channel. The images were displayed on 2 separate screens, one for the video endoscope-derived images and 


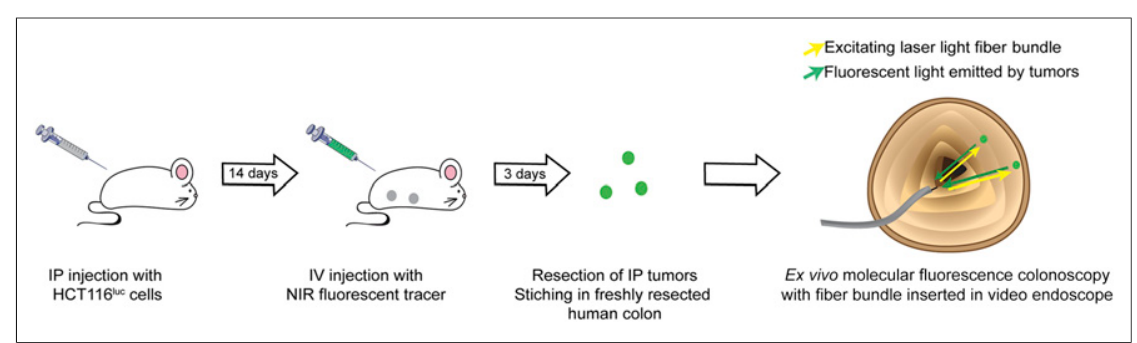

FIGURE 1. HCT116luc tumor cells were inoculated intraperitoneally (IP) in athymic nude mice. After tumor establishment, targeted NIR fluorescent tracer (bevacizumab-800CW or cetuximab$800 \mathrm{CW}$ ), nontargeted NIR fluorescent tracer (IgG-800CW), or sodium chloride was administered intravenously (IV). Three days after administration, intraperitoneal tumor lesions (diameter, 2-5 mm) were harvested and stitched in freshly resected human colon. Ex vivo molecular fluorescence colonoscopy procedure was performed using standard video endoscope, with the fiber bundle of NIR fluorescence endoscopy platform passed through the working channel.

the other for the composite images of the fiber bundle, combining color and fluorescence. A qualitative assessment of tumor visualization was made during endoscopy, and video footage and photo material were collected.

Ex Vivo Analyses. To further validate the findings of the simulated endoscopy procedure, the remaining harvested HCT116 $6^{\text {luc }}$ human xenograft tumors, mouse organs, and a part of the human colon tissue were formalin-fixed and paraffin-embedded for microscopic analysis. To detect whether 800-nm fluorescence signals were present, $4-\mu \mathrm{m}$ slides were obtained, deparaffinized (10 min xylene), scanned on an Odyssey Infrared Imaging System (intensity 5; LI-COR Biosciences), and subsequently stained with hematoxylin and eosin. Hoechst staining (33258; Invitrogen) was used to visualize nuclei. Fluorescence ;microscopy was performed using a Leica DM6000B inverted wide-field microscope $(63 \times$ magnification, immersion oil), with a mercury short-arc reflector lamp (HXP-R120W/45C VIS; Leica), DFC365FX camera (Leica), and a filter set (49037ET; Chroma Technology). Images were processed with LAS-AF2 software (Leica Microsystems).

\section{Statistical Analysis}

Statistical analysis was performed using SPSS Statistics 20 (IBM). Per tissue type, the difference in staining intensity between aberrant tissue and adjacent normal tissue was determined via nonparametric Mann-Whitney $U$ testing. The correlation between histologic stage of Lynch tissue (LGD adenomas, HGD adenomas, and cancer tissue) and staining intensity (0-3) was tested using a Kruskal-Wallis test, corrected

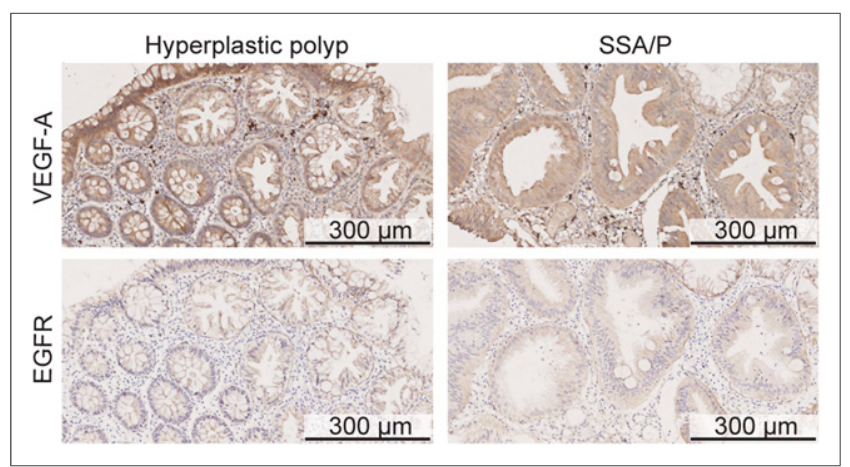

FIGURE 2. (A) Representative immunohistochemistry stainings of VEGF-A and EGFR in $1 \mathrm{HP}$ and $1 \mathrm{SSA} / \mathrm{P}$. Both lesions were scored 3 (strong) for VEGF-A and 2 (moderate) for EGFR. for multiple testing. Data are presented using Prism (version 5; GraphPad Software) and Adobe Illustrator CS6.

\section{RESULTS}

\section{High VEGF-A Expression in Human Colorectal Lesions}

Immunohistochemistry for VEGF-A showed a homogeneous staining pattern within dysplastic and carcinomatous areas (Fig. 2; Supplemental Fig. 1A [supplemental materials are available at http:// jnm.snmjournals.org]). From normal colon crypts toward dysplastic and cancerous areas, a gradually increasing staining intensity was observed (Supplemental Fig. 1B). Moderate to strong VEGF-A expression was observed in $96 \%$ of Lynch LGD adenomas, 79\% of Lynch HGD adenomas, $94 \%$ of Lynch colon cancer tissue, and $94 \%$ of SSA/P. No significant differences in staining intensities could be observed between the different histologic stages (LGD, HGD, carcinoma) of the LS samples. In addition, VEGF-A was found to be a relevant target for other polyps as well, showing a 94\% VEGF-A expression in HGD sporadic adenomas and $95 \%$ in HP lesions (Fig. 3). For all adenomas, the VEGF-A expression was significantly higher than the adjacent normal colonic crypts $(P<0.001)$, signifying the potential of this target for adenoma detection.

The percentage of EGFR-positive samples was lower for all tissue types: $52 \%$ of Lynch LGD adenomas, $51 \%$ of Lynch HGD adenomas, $68 \%$ of Lynch cancer samples, $52 \%$ of SSA/P lesions, $51 \%$ of sporadic HGD adenomas, and $58 \%$ of HP lesions showed a positive receptor staining (Fig. 3). In contrast to VEGF-A, EGFR immunohistochemistry results showed a heterogeneous expression pattern throughout the neoplastic lesions, resulting in both EGFRnegative and EGFR-positive crypts within 1 adenoma (Fig. 2; Supplemental Fig. 1A). The EGFR expression was significantly higher in neoplastic lesions than in the adjacent normal colon crypts $(P<0.001)$. A gradient of increased EGFR expression from normal toward dysplastic crypts was not observed.

\section{Performance of NIR Fluorescence Endoscopy Platform}

The resolution of the fluorescence endoscopy platform was characterized by imaging the USAF 1951 resolution test chart with the system's color channel (Fig. 4A). Here we can distinctly identify the vertical and horizontal lines in element 3 of group 1, which translates to a spatial resolution of $198.42 \mu \mathrm{m}$ at a distance of $2 \mathrm{~cm}$. The signal-to-noise ratios were determined for a dilution series of IRDye $800 \mathrm{CW}$ with concentrations ranging between $26.05 \mu \mathrm{M}$ and $1.55 \mathrm{pM}$ (Fig. 4B). Each subsequent dilution was obtained of a halved concentration of IRDye800CW dissolved in PBS. The detection limit was defined as the concentration in which the signal is 3 times higher than the noise, which corresponds to a signal-to-noise ratio of $9.5 \mathrm{~dB}$. The detection limit, calculated from the regression line, lies at a concentration of $19.80 \mathrm{nM}$.

Ex Vivo Colonoscopy Procedure: Real-Time Visualization of NIR Fluorescent Lesions. During the ex vivo colonoscopy procedure, the NIR fluorescence endoscopy platform exhibited sufficient sensitivity and resolution for the visualization of all small HCT116 $6^{\text {luc }}$ tumors that were labeled with bevacizumab-800CW and 


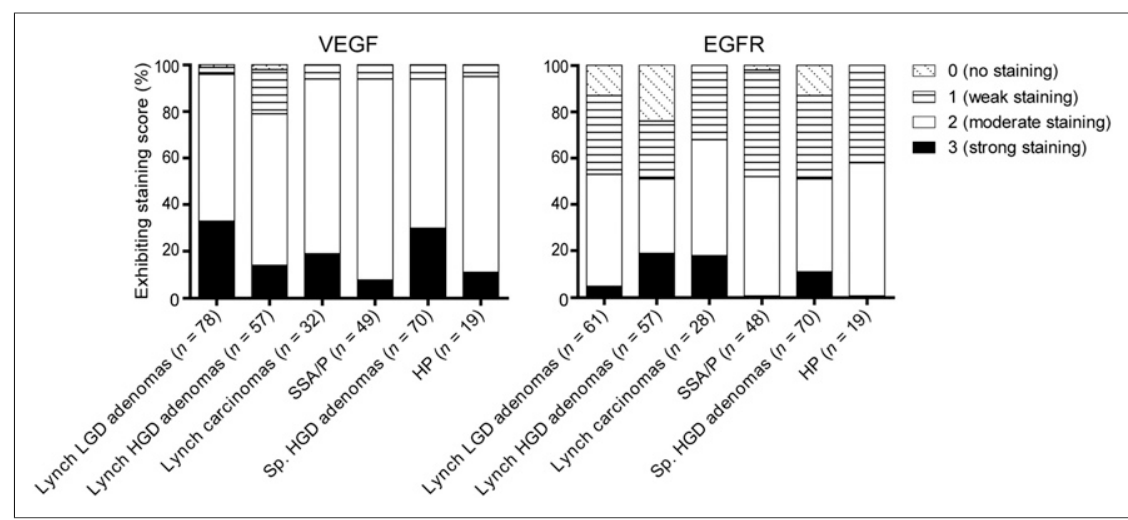

FIGURE 3. VEGF-A and EGFR expression for colorectal adenomas with LGD of patients with LS, adenomas with HGD of patients with LS, carcinomas of patients with LS, SSA/P, sporadic (Sp.) adenomas with HGD, and HPs.

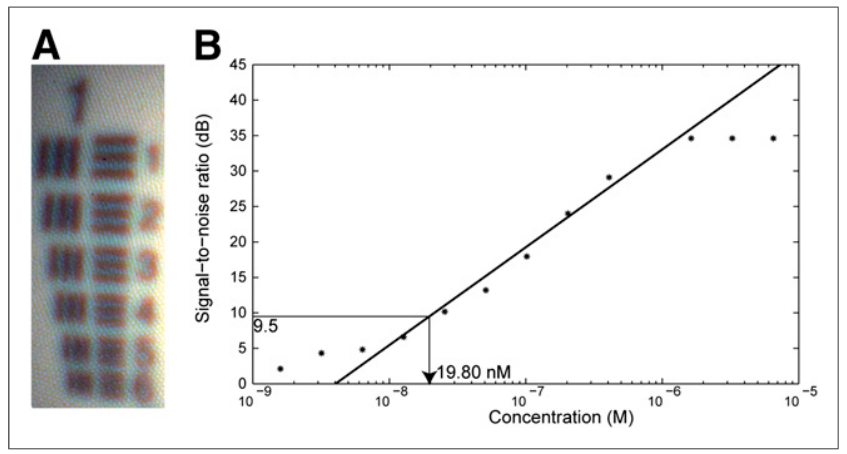

FIGURE 4. (A) Detail of USAF 1951 resolution target image, in which vertical and horizontal lines in element 3 of group 1 can be distinctly identified, which translates to spatial resolution of $198.42 \mu \mathrm{m}$ at distance of $2 \mathrm{~cm}$. (B) Signal-to-noise ratio over IRDye800CW concentration measured from dilution series. Detection limit of $9.5 \mathrm{~dB}$ lies at concentration of $19.80 \mathrm{nM}$.

cetuximab-800CW (Fig. 5; Supplemental Fig. 2; Supplemental Video 1). The endoscopist was able to instantly detect the specifically targeted tumors $(2-5 \mathrm{~mm})$ and differentiate these from the control tumors. White-light, fluorescence, and composite images were displayed in real time and with a wide field of view. Fluorescence was also clearly visible when the fiber was retracted to a larger distance $(\sim 5 \mathrm{~cm})$ of the fluorescent tumors. Control tumors from mice that were given $\mathrm{IgG}-800 \mathrm{CW}$ or sodium chloride showed negligible fluorescence signals, and there was no interference of autofluorescence of the human colon tissue.

\section{IRDye800CW-Labeled Tracers: Ex Vivo Established Target Specificity}

Odyssey Imaging System and Fluorescence Microscopy. Ex vivo macroscopic NIR fluorescence imaging of the deparaffinized tissue slides revealed highly fluorescent tumors with clear tumor delineation (Fig. 6B; Supplemental Fig. 3B) for both bevacizumab$800 \mathrm{CW}$ and cetuximab-800CW. IgG- $800 \mathrm{CW}$ and negative tumors showed low autofluorescence signals (Supplemental Fig. $3 \mathrm{E})$. NIR fluorescence was low in the hematoxylin and eosinconfirmed necrotic areas of the tumors and healthy adjacent mouse tissue (Fig. 6A; Supplemental Figs. 3A and 3D, orange arrows). Fluorescence microscopy confirmed the localization of
bevacizumab-800CW and cetuximab-800CW in the vital parts of the HCT116 $6^{\text {luc }}$ tumors (Fig. 6C; Supplemental Fig. 3C). For bevacizumab-800CW, the fluorescent signal was mainly located in the tumor stroma and surrounding the tumor blood vessels, corresponding with our previous observations for ${ }^{89} \mathrm{Zr}$ - and ${ }^{111}$ In-labeled bevacizumab (14,21). A more homogeneous distribution was seen for cetuximab-800CW in the tumor lesions, with membranous and cytoplasmic localization of the NIR fluorescence signals (22).

\section{DISCUSSION}

This study demonstrated, in a simulation model, that it is feasible to visualize colorectal lesion in real time, using a novel fluorescence endoscopy platform combined with VEGF-A- and EGFR-targeting NIR fluorescent tracers. Given the observed overall VEGF-A overexpression in colorectal lesions, this target appears to be the most relevant for molecular colorectal screening purposes. Especially in colorectal lesions that are easily missed during endoscopy, such as SSA/P lesions and Lynch adenomas, VEGF-A was highly overexpressed. The ability to visualize colon lesions in the simulation model used in this study, in combination with the good-manufacturing-practice production of $800 \mathrm{CW}$-labeled antibodies, allows rapid translation of this technique toward the clinic.

To the best of our knowledge, no prior data are available on VEGF-A expression in SSA/P or neoplastic lesions from high-risk patients, such as those with LS. We found a moderate to strong VEGF-A expression in most SSA/P and Lynch adenomas. The high VEGF-A expression in sporadic adenomas was in concordance with the literature $(12,13)$. VEGF-A expression was significantly higher in adenoma crypts than in adjacent normal colon crypts, which is a key requirement for successful visualization of target lesions by molecular imaging. Moreover, we observed a gradually increased staining intensity from normal colon crypts toward dysplastic and cancerous areas in many of the samples, which can be explained by the fact that VEGF-A expression is regulated by several growth factors present in the microenvironment of premalignant and malignant lesions (23).

In contrast, EGFR is overexpressed in only approximately $50 \%$ of all adenoma lesions, including those of LS patients, and was expressed more heterogeneously throughout the lesions. The observed EGFR expression is in line with previous findings in sporadic adenomas (24). Therefore, VEGF-A seems the most suitable target for screening purposes. In contrast, cetuximab-800CW may still be valuable in evaluating the EGFR expression status of an already identified lesion. In this setting, our approach could play an assisting role in molecular treatment decision-making processes, whereas EGFR-targeting therapeutics are currently being applied in colorectal carcinomas.

The use of a fiber bundle-based approach is relatively inexpensive and can easily be incorporated in standard clinical endoscopy procedures, because the fiber bundle fits through the working channel of a routine clinical video endoscope. Although the fiber images have a significantly lower resolution than highdefinition video endoscope images, the molecularly guided approach is sensitive and provides strong contrast between the NIR 


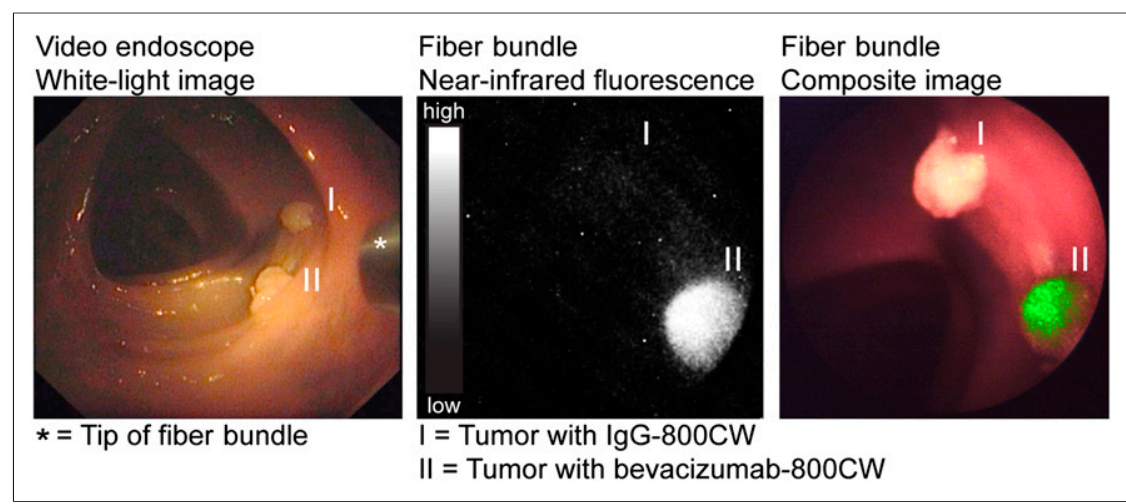

FIGURE 5. Images acquired during ex vivo molecular fluorescence colonoscopy of IgG-800CW (I) and bevacizumab-800CW (II) targeted tumors ( $3 \times 3 \mathrm{~mm}$ in size). Endoscopy images were obtained with video endoscope and fiber bundle. White-light, fluorescence and composite images of fiber bundle were real-time-projected.

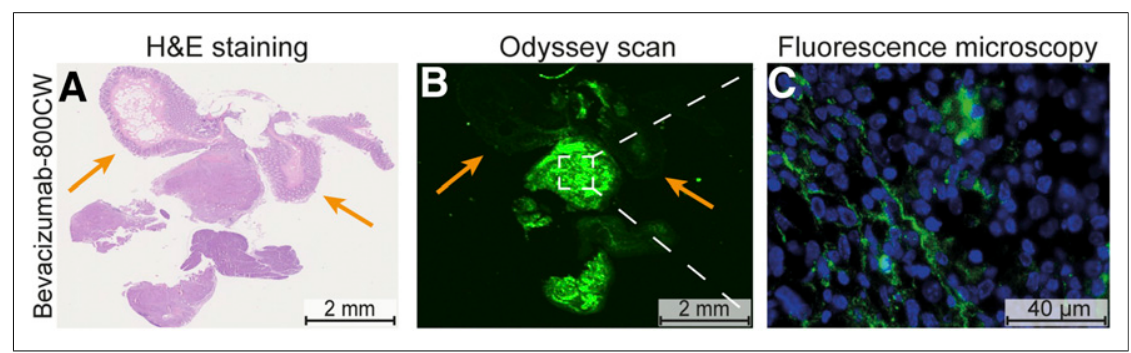

FIGURE 6. Hematoxylin and eosin (H\&E) staining (A), corresponding fluorescence image obtained with Odyssey Scanner $(B)$, and corresponding fluorescence microscopy image $(C)$ of HCT116luc tumor targeted with bevacizumab-800CW. Odyssey scan shows clear fluorescence in all tumor tissue at $800 \mathrm{~nm}$, whereas uptake was negligible in histologic normal tissue (colon and muscle of mice, orange arrows). Fluorescence microscopy showed bevacizumab-800CW to be mainly localized in stroma of tumors.

fluorescent-targeted lesions and the surrounding normal tissue. Because the images are in real time and with a wide field of view, this approach can assist in the screening of large surfaces, with the white-light high-definition endoscope providing morphologic orientation while the fiber can support as a red-flag method.

The technique described in this study has several advantages when compared with other molecular fluorescence endoscopy approaches. First, confocal laser endomicroscopy has shown promising results both preclinically and clinically but has a limited field of view (25-27). Therefore, confocal laser endomicroscopy is not suitable for screening purposes, as our technique is, but rather a tool for lesion characterization. Second, other research groups have described topical spraying of tracer products to improve detection of dysplastic regions, but this is most attractive for relatively small areas and therefore not practical in the colon (28-30). An approach using intravenously injected tracers can circumvent these issues. Finally, the use of a fluorescent tracer emitting in the NIR light spectrum likely improves specificity and tumor-to-background signals when compared with fluorescent dyes of the visible spectrum, because autofluorescence is negligible in the NIR range (Supplemental Figs. 4 and 5) (31).

Previously, in an HCT116 ${ }^{\text {luc }}$ xenograft model, a tumor-to-background ratio of $3.2 \pm 0.9$ was seen for bevacizumab-800CW and $5.7 \pm 3.0$ for cetuximab-800CW $3 \mathrm{~d}$ after injection (Supplemental Fig. 4). However, the commonly used tumor-to-background ratio cannot be reliably evaluated in mice, because the tracers are targeted toward human VEGF-A and EGFR. As a consequence, the used artificial model, targeted intraperitoneal xenograft tumors stitched in a human colon specimen, demonstrates the practical feasibility of the proposed technique, but no statements can be made regarding specific tumor or adenoma accumulation in comparison to uptake in healthy human colon tissue. However, clinical studies evaluating the use of ${ }^{89} \mathrm{Zr}$-labeled bevacizumab in breast and kidney cancer patients did not show aspecific accumulation of the tracer in the colon (32). Also, the significantly higher VEGF-A expression in colorectal adenomas than in adjacent normal tissue implies that bevacizumab-800CW could be a promising tracer from a diagnostic point of view. The initiated clinical studies (NCT01972373 and NCT02113202) should give insight into the in vivo sensitivity and specificity of bevacizumab-800CW toward VEGF-A in different colorectal lesions, as well as the safety and optimal tracer dose to perform the molecular fluorescence endoscopy procedure.

\section{CONCLUSION}

NIR molecular fluorescence endoscopy is a promising technique that allows realtime, wide-field visualization of both tissue morphology and molecular characteristics. In this study, we demonstrated that our good manufacturing practice-produced NIR tracers, targeting VEGF and EGFR, could be clearly visualized during a simulated molecular fluorescence endoscopy procedure. On the basis of the expression profiles observed in a large set of different colorectal samples, including those in highrisk patients with SSA/P and LS, VEGF-A seems a suitable target for molecular fluorescence endoscopic screening purposes. Clinical studies have been initiated and are currently recruiting patients to validate the potential of this technology during colonoscopy (clinicaltrials.gov: NCT01972373 and NCT02113202).

\section{DISCLOSURE}

The costs of publication of this article were defrayed in part by the payment of page charges. Therefore, and solely to indicate this fact, this article is hereby marked "advertisement" in accordance with 18 USC section 1734. The research leading to these results was partially supported by the European Union under the grant agreement ERC-OA-2012-PoC-324627 and partially supported by the Dutch Cancer Society, RUG 2012-5416. No other potential conflict of interest relevant to this article was reported.

\section{REFERENCES}

1. Rockey DC, Paulson E, Niedzwiecki D, et al. Analysis of air contrast barium enema, computed tomographic colonography, and colonoscopy: prospective comparison. Lancet. 2005;365:305-311. 
2. Heresbach D, Barrioz T, Lapalus M, et al. Miss rate for colorectal neoplastic polyps: a prospective multicenter study of back-to-back video colonoscopies. Endoscopy. 2008;40:284-290.

3. Stoffel EM, Turgeon DK, Stockwell DH, et al. Missed adenomas during colonoscopic surveillance in individuals with lynch syndrome (hereditary nonpolyposis colorectal cancer). Cancer Prev Res (Phila). 2008;1:470-475.

4. Mecklin JP, Aarnio M, Läärä E, et al. Development of colorectal tumors in colonoscopic surveillance in lynch syndrome. Gastroenterology. 2007;133:1093-1098.

5. Rijcken FEM, Hollema H, Kleibeuker JH. Proximal adenomas in hereditary nonpolyposis colorectal cancer are prone to rapid malignant transformation. Gut. 2002;50:382-386.

6. Corley DA, Jensen CD, Marks AR, et al. Adenoma detection rate and risk of colorectal cancer and death. N Engl J Med. 2014;370:1298-1306.

7. East JE, Suzuki N, Stavrinidis M, Guenther T, Thomas HJW, Saunders BP. Narrow band imaging for colonoscopic surveillance in hereditary non-polyposis colorectal cancer. Gut. 2008;57:65-70.

8. Ramsoekh D, Haringsma J, Poley JW, et al. A back-to-back comparison of white light video endoscopy with autofluorescence endoscopy for adenoma detection in high-risk subjects. Gut. 2010;59:785-793.

9. Chung SJ, Kim D, Song JH, et al. Comparison of detection and miss rates of narrow band imaging, flexible spectral imaging chromoendoscopy and white light at screening colonoscopy: a randomised controlled back-to-back study. Gut. 2014;63:785-791.

10. de Vries EGE, Munnink THO, van Vugt MATM, Nagengast WB. Toward molecular imaging-driven drug development in oncology. Cancer Discov. 2011;1:25-28.

11. Hoetker MS, Goetz M. Molecular imaging in endoscopy. United European Gastroenterol J. 2013;1:84-92.

12. Staton CA, Chetwood ASA, Cameron IC, Cross SS, Brown NJ, Reed MWR. The angiogenic switch occurs at the adenoma stage of the adenoma carcinoma sequence in colorectal cancer. Gut. 2007;56:1426-1432.

13. Hanrahan V, Currie MJ, Gunningham SP, et al. The angiogenic switch for vascular endothelial growth factor (VEGF)-A, VEGF-B, VEGF-C, and VEGF-D in the adenoma-carcinoma sequence during colorectal cancer progression. $J$ Pathol. 2003;200:183-194.

14. Terwisscha van Scheltinga AG, van Dam GM, Nagengast WB, et al. Intraoperative near-infrared fluorescence tumor imaging with vascular endothelial growth factor and human epidermal growth factor receptor 2 targeting antibodies. $\mathrm{J} \mathrm{Nucl}$ Med. 2011;52:1778-1785.

15. Cohen R, Stammes MA, de Roos IH. Inert coupling of IRDye800CW to monoclonal antibodies for clinical optical imaging of tumor targets. EJNMMI Res. 2011;1:31.

16. Marshall MV, Draney D, Sevick-Muraca EM, Olive DM. Single-dose intravenous toxicity study of IRDye $800 \mathrm{CW}$ in Sprague-Dawley rats. Mol Imaging Biol. 2010;12:583-594.

17. Zinn KR, Korb M, Samuel S, et al. IND-directed safety and biodistribution study of intravenously injected cetuximab-IRDye800 in cynomolgus macaques. Mol Imaging Biol. 2015;17:49-57.
18. van Dam GM, Themelis G, Crane LMA, et al. Intraoperative tumor-specific fluorescence imaging in ovarian cancer by folate receptor- $\alpha$ targeting: first inhuman results. Nat Med. 2011;17:1315-1319.

19. Themelis G, Yoo JS, Soh K-S, Schulz R, Ntziachristos V. Real-time intraoperative fluorescence imaging system using light-absorption correction. J Biomed Opt. 2009;14:064012.

20. Garcia-Allende PB, Glatz J, Koch M, et al. Towards clinically translatable NIR fluorescence molecular guidance for colonoscopy. Biomed Opt Express. 2013;5:78-92.

21. Nagengast WB, de Vries EG, Hospers GA, et al. In vivo VEGF imaging with radiolabeled bevacizumab in a human ovarian tumor xenograft. J Nucl Med. 2007;48:1313-1319.

22. Eiblmaier M, Meyer LA, Watson MA, Fracasso PM, Pike LJ, Anderson CJ. Correlating EGFR expression with receptor-binding properties and internalization of ${ }^{64} \mathrm{Cu}$-DOTA-cetuximab in 5 cervical cancer cell lines. $\mathrm{J} \mathrm{Nucl} \mathrm{Med}$. 2008;49:1472-1479.

23. Ferrara N. Vascular endothelial growth factor: basic science and clinical progress. Endocr Rev. 2004;25:581-611.

24. Bansal A, Liu X, McGregor DH, Singh V, Hall S. Correlation of epidermal growth factor receptor with morphological features of colorectal advanced adenomas: a pilot correlative case series. Am J Med Sci. 2010;340:296-300.

25. Neumann H, Kiesslich R, Wallace MB, Neurath MF. Confocal laser endomicroscopy: technical advances and clinical applications. Gastroenterology. 2010;139:388392.

26. Goetz M, Ziebart A, Foersch S, et al. In vivo molecular imaging of colorectal cancer with confocal endomicroscopy by targeting epidermal growth factor receptor. Gastroenterology. 2010;138:435-446.

27. Sturm MB, Joshi BP, Lu S, et al. Targeted imaging of esophageal neoplasia with a fluorescently labeled peptide: first-in-human results. Sci Transl Med. 2013;5: $184 \mathrm{ra61.}$

28. Joshi BP, Miller SJ, Lee CM, Seibel EJ, Wang TD. Multispectral endoscopic imaging of colorectal dysplasia in vivo. Gastroenterology. 2012;143:1435-1437.

29. Miller SJ, Joshi BP, Feng Y, Gaustad A, Fearon ER, Wang TD. In vivo fluorescencebased endoscopic detection of colon dysplasia in the mouse using a novel peptide probe. PLoS One. 2011;6:e17384.

30. Bird-Lieberman EL, Neves AA, Lao-Sirieix P, et al. Molecular imaging using fluorescent lectins permits rapid endoscopic identification of dysplasia in Barrett's esophagus. Nat Med. 2012;18:315-321.

31. Burggraaf J, Kamerling IMC, Gordon PB, et al. Detection of colorectal polyps in humans using an intravenously administered fluorescent peptide targeted against c-Met. Nat Med. 2015;21:955-961.

32. Oosting SF, Brouwers AH, van Es SC, et al. ${ }^{89} \mathrm{Zr}$-bevacizumab PET visualizes heterogeneous tracer accumulation in tumor lesions of renal cell carcinoma patients and differential effects of antiangiogenic treatment. J Nucl Med. 2015;56: 63-69. 